\title{
Molecular detection and identification of Leishmania infection in naturally infected sand flies in a focus of cutaneous leishmaniasis in northern Morocco
}

Nargys Es-Sette ${ }^{1,2}$, Malika Ajaoud ${ }^{1}$, Abderrahman Laamrani-Idrissi ${ }^{3}$, Fouad Mellouki ${ }^{2}$ and Meryem Lemrani ${ }^{1 *}$

\begin{abstract}
Background: Cutaneous leishmaniasis is an infectious disease caused by various species of the flagellate protozoan Leishmania. During the past 20 years, cutaneous leishmaniasis has emerged as a major public health threat in Morocco. The main objective of this study was to study the occurrence of Leishmania infection in vectors and to identify sand fly blood meal sources in an endemic locality of cutaneous leishmaniasis within Sefrou province, where the vectors of leishmaniasis were still unknown.

Methods: 2650 sand flies were collected using CDC miniature light traps and identified morphologically. The identified sand flies were tested for Leishmania infection by nested PCR. The source of blood meal of 10 freshly engorged females: 6 Phlebotomus longicuspis and 4 Phlebotomus sergenti, was determined using the Cyt $b$ sequence.

Results: The collected sand flies consisted of 10 species, seven of which belonged to the genus Phlebotomus and three to the genus Sergentomyia. The most abundant species was P. longicuspis, accounting for $72 \%$ of the total sand flies collected. In females of three P. Iongicuspis and four P. sergenti, Leishmania infantum and Leishmania tropica DNA was detected, respectively.

The source of blood meal of engorged females showed that all sand flies tested fed on humans.

Conclusions: We report for the first time the natural infection of $P$. Iongicuspis with $L$. infantum in Morocco. The high frequency of this species in this region, in addition to its anthropophilic character make $P$. longicuspis the putative vector of $L$. infantum in this cutaneous leishmaniasis focus where $L$. tropica is confirmed as the causative agent of the disease and $P$. sergenti as its vector. The presence of $L$. infantum, and its presumed vector in this area, makes this a site of high risk of visceral leishmaniasis, mostly because of the proximity of a focus of human and canine visceral leishmaniasis.
\end{abstract}

Keywords: P. sergenti, P. longicuspis, L. tropica, L. infantum, Cutaneous leishmaniasis, Blood meal, Morocco

\footnotetext{
*Correspondence: meryem.lemrani@gmail.com

${ }^{1}$ Laboratoire de Parasitologie et Maladies Vectorielles, Institut Pasteur du

Maroc, 1 Place Louis Pasteur, 20360 Casablanca, Morocco

Full list of author information is available at the end of the article
} 


\section{Background}

Morocco lies in the Mediterranean region, where two clinico-epidemiological forms of leishmaniasis are endemic: visceral leishmaniasis (VL) and cutaneous leishmaniasis (CL). There were 9,000 CL cases reported in 2010 compared to 3,361 in 2006 and 655 in 1998, showing a clear tendency for an increase over the last decade [1]. Three species of Leishmania cause CL: L. major, L. tropica and less frequently $L$. infantum. Zoonotic CL caused by L. major is endemic especially in the southern slopes of the Atlas Mountains where its unique vector, $P$. papatasi and its reservoir host, Meriones shawi, are prevalent [2]. Anthroponotic CL due to L. tropica is endemic in arid and semi-arid regions in the center and mainly on the north western slopes of the Atlas Mountains. Its proven vector is P. sergenti [3].

Cutaneous leishmaniasis due to L. infantum occurs sporadically in the north of Morocco as reported by many authors [4-6]. L. infantum is also responsible for widespread visceral leishmaniasis in the North-Eastern slope of the Rif Mountains, the most active area of this form of the disease. The dog is the main domestic reservoir of the species [7]. Among about 20 sand fly species that play a significant role in the transmission of Leishmania parasites in the Mediterranean basin, members of the subgenus Larroussius represent the most important vectors of L. infantum [8-10]. In Morocco P. perniciosus and P. longicuspis were suspected as vectors of $L$. infantum based solely on their abundance in active foci of leishmaniasis, however, one specimen of $P$. ariasi caught inside a house in a focus of visceral leishmaniasis in northern Morocco was found to be naturally infected with promastigotes identified as $L$. infantum by immunofluorescence with monoclonal antibodies [11].

In northern Morocco, Sefrou province has been established as a focus of cutaneous and visceral leishmaniasis. According to the local health centers, sporadic human cases continue to occur every year in several localities of this province. The first CL cases appeared in 1997, the epidemiological situation remained stable until the year 2000. From 2000 to 2011, an average of 67 CL cases per year was recorded [1].

In 2008, the Moroccan Ministry of Health reported 9 VL cases and 64 CL cases spread over several sites belonging to Sefrou province, the causative agents of CL and VL were identified as L. tropica [12] and L. infantum, respectively $[7,13,14]$. Despite the large number of foci of $\mathrm{CL}$ and VL in this province, the vectors have never been identified with certainty.

The incrimination of a sand fly species as being the vector of Leishmania species is complicated; Killick Kendrick [9] suggested the following criteria for incrimination of a vector sand fly: anthropophilic behavior and common infection with the same Leishmania parasite as that found in man in the same place. Further evidence such as the demonstration that the fly feeds regularly on the reservoir host, concordance in the distribution of the fly and the disease in man, proof that the parasite develops in infected flies and the experimental transmission of the parasite by the bite of the fly can reinforce the incrimination.

The classical method of Leishmania detection requires the dissection of freshly caught individual sand flies and culture of parasites found in the sand fly gut $[15,16]$, but this technique is time-consuming, needs dissecting expertise and a large number of specimens, since the Leishmania infection rate in sand flies is usually very low even in highly endemic areas [17]. In recent years, molecular methods are increasingly employed in epidemiological studies to detect infection and to characterize Leishmania parasites in hosts and vectors; they are highly sensitive in the detection of Leishmania spp. infections in phlebotomines $[18,19]$. This sensitivity increases the understanding of vector competence and leishmaniasis epidemiology $[18,19]$. Accurate and sensitive diagnostic and identification procedures are also required to distinguish Leishmania species whose geographic distribution can overlap, which is crucial for appropriate public health control measures.

Hence, the objective of this work was to identify the species of phlebotomine sand flies involved in an endemic locality of cutaneous leishmaniasis within Sefrou province, where the vectors of leishmaniasis were still unknown. Molecular tools were used for the first time in the identification of the host blood feeding preferences of sand flies, together with the detection of parasitic infection within sand flies.

\section{Methods}

\section{Study sites and collection of sand flies}

Sand fly sampling was carried out from June-August 2008 (during the period of peak sand fly activity) in Louata, a locality situated in Sefrou province in northern Morocco (Figure 1). It is located in the north-west of the middle Atlas Mountain. Louata supports a semi-arid climate with mean annual rainfall of about $450 \mathrm{~mm}$. The average elevation of Louata is 589 meters. Sand flies were collected by CDC light traps placed in or near houses or animal housing facilities. Traps were suspended so that the attracting light source was approximately 1.5 meters above the ground and were set in the late afternoon with collection occurring the following morning. The sand flies were then placed in $1.5 \mathrm{ml}$ Eppendorf tubes and preserved at $-80^{\circ} \mathrm{C}$. Phlebotomine specimens of both genders were identified by their morphological characteristics. Each sand fly was dissected under a binocular microscope, on a sterilized microscopic slide using sterile steel entomological needles. The head and genitalia of each sand fly were mounted 


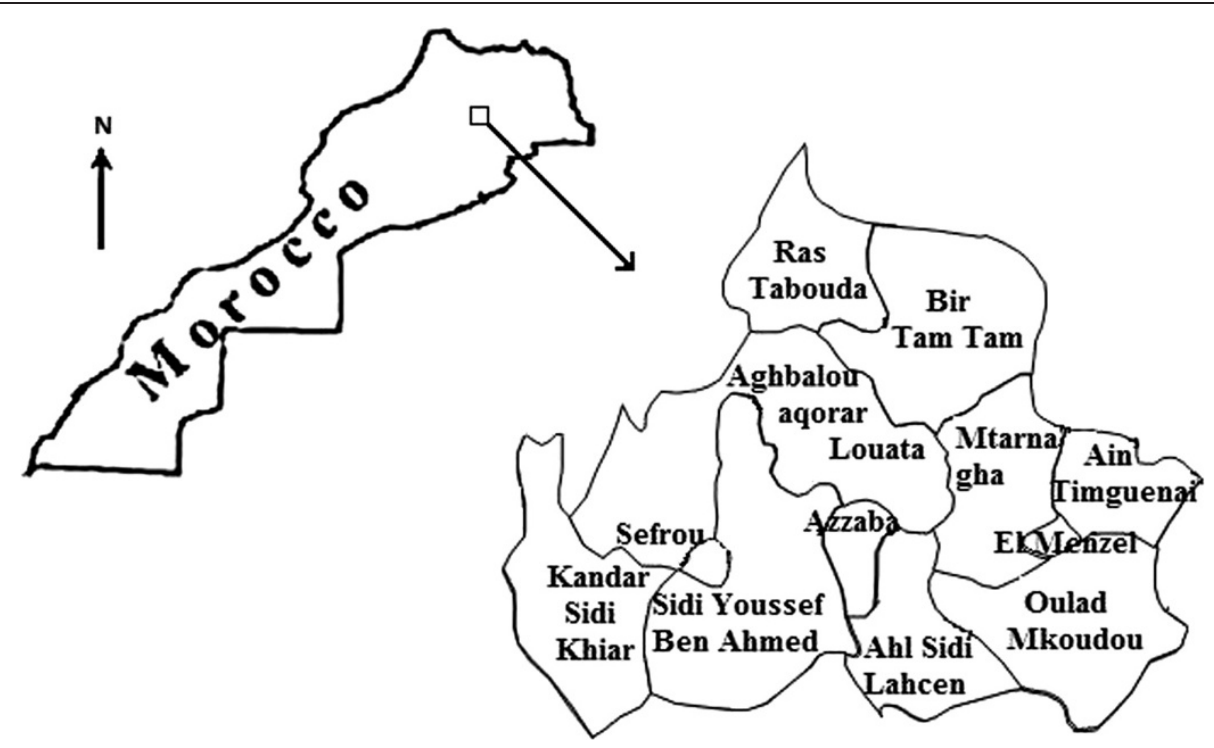

Figure 1 Map of Morocco showing Sefrou province and the Louata locality, the site of the survey.

under a cover slip in Marc-André solution for morphological identification at the species level according to morphological keys described by the Moroccan Health Ministry [20]. After dissection, the abdomen and the thorax of each female specimen were transferred to sterile $1.5 \mathrm{ml}$ Eppendorf tube. They were subsequently grouped into 55 pools according to date, collection site and species, with up to 30 specimens in each pool and were stored at $-20^{\circ} \mathrm{C}$. The preserved pools were processed for DNA extraction. For each female, it was determined whether it was engorged or unfed.

\section{Detection of Leishmania spp. in female sand flies}

For each pool, DNA extraction was performed by the phenol-chloroform method [21]. DNA was purified by Qiagen kit according to the instructions of the manufacturer. Female sand flies were screened for Leishmania infection by nested PCR. Positive PCR were followed by direct sequencing.

\section{Nested PCR for amplifying the ITS-5.8S rDNA gene of Leishmania species}

Each PCR was carried out in two separate tubes [22,23]. The first-stage PCR used the forward primer IR1 (5' GC TGTAGGTGAACCTGCAGCAGCTGGATCATT 3', at the 3 ' end of the SSU rRNA gene) with the reverse primer IR2 (5' GCGGGTAGTCCTGCCAAACACTCAGGTCTG 3', at the 5 ' end of the large subunit rRNA gene).

The ITS1-5.8S rDNA gene was amplified using the nested forward primer ITS1F (5' GCAGCTGGATCAT TTTCC 3'; overlapping the 3' end of the SSU rRNA gene and ITS1) with the nested reverse primer ITS2R4
(5' ATATGCAGAAGAGAGGAGGC 3'; at the 5' end of ITS2) [23].

The first amplification reaction totaled $20 \mu \mathrm{l}$, containing 1xTaq polymerase buffer B (Invitrogen), $1.5 \mathrm{mM} \mathrm{MgCl}_{2}$, $60 \mu \mathrm{M}$ each dNTP, $1 \mu \mathrm{M}$ primer IR1, $1 \mu \mathrm{M}$ primer IR2, 1 unit Taq polymerase (Invitrogen). The mixture was incubated in a thermocycler involving an initial denaturation at $94^{\circ} \mathrm{C}$ for $3 \mathrm{~min}$, followed by 37 cycles each consisting of three steps: $30 \mathrm{~s}$ at $94^{\circ} \mathrm{C}$ (denaturation), $30 \mathrm{~s}$ at $58^{\circ} \mathrm{C}$ (annealing) and $90 \mathrm{~s}$ at $72^{\circ} \mathrm{C}$ (extension). After the last cycle, the extension step was continued for a further $10 \mathrm{~min}$, and then the reaction was held at $4^{\circ} \mathrm{C}$.

The nested amplification was carried out in a second tube, with the reaction mix again totaling $20 \mu \mathrm{l}$ and containing the same reagents as the first stage, except that the primers were now $1 \mu \mathrm{M}$ primer ITS1F and $1 \mu \mathrm{M}$ primer ITS2R4, and the target DNA was provided by adding $1 \mu \mathrm{l}$ of the completed first-stage PCR reaction. Cross-contamination was monitored by negative controls for sample extraction and PCR solutions. The thermocycler program was as described for the first-stage.

The final PCR products of 462 bp were directly sequenced to identify Leishmania species infecting individual sand flies. They were purified using the Exonuclease I/Shrimp Alkaline Phosphatase (GE Healthcare, US) before sequencing by using a BigDye Terminator version 3.1 Cycle Sequencing kit (Applied Biosystems, Foster City, CA, USA) and an ABI PRISM 3130 DNA automated sequencer (Applied Biosystems). Sequencing data were analyzed using SeqScape v.2.5 software (Applied Biosystems). Sequences were aligned using the multiple alignment program MEGA 6. A phylogenetic tree was constructed by using the Neighbor-Joining method in agreement with the 
Kimura 2-parameter model with uniform rates for transitions and transversions. Bootstrap replicates were performed to estimate node reliability, and values were obtained from 1,000 randomly selected samples of the aligned sequence data. Sequences were compared with entries retrieved from GenBank.

\section{Leishmania DNA sequencing and Neighbor-Joining analysis}

The sequences obtained (KJ567476, KJ567477, KJ567478, KJ567479, KJ567480, KJ567481, KJ567482) were initially analyzed by the BLAST program; 3 P. longicuspis and 4 $P$. sergenti pools were found to be infected by $L$. infantum and L. tropica respectively (Table 1 ). The sequencing results were processed using MEGA software version 6 [24] and the neighbor joining (NJ) method [25] was used to construct the phylogenetic tree. The percentage of replicate trees in which the associated taxa clustered together in the bootstrap test (1000 replicates) are shown next to the branches [26]. All nucleotide sequences obtained in this study were compared using the program ClustalX with sequences from L. infantum and L. tropica retrieved from GenBank.

\section{Blood meal identification}

Genomic DNA was extracted from 10 fully engorged sand flies (6 P. longicuspis and 4 P. sergenti) using the QiaAmp blood DNA mini Kit (Qiagen, Hilden, Germany, Cat. no. 51106), as per the manufacturer's instructions. The DNA was eluted in $0.1 \mathrm{~mL}$ of $\mathrm{AE}$ buffer (supplied with the Qiagen kit) after extraction.

DNA of hosts was amplified using PCR with universal primers complementary to the conserved region of mitochondrial DNA (mt DNA) Cyt $b$ gene. The primers, L14841 (F5' CCATCCAACATCTCAGCATGATGAAA 3') and H15149 (R5' CCCCTCAGAATGATATTTGTCCT CA 3') amplified 359 bp of the Cyt $b$ gene [27].
A mixture of $25 \mu \mathrm{L}$ solution containing $1 \times$ Taq polymerase buffer $\mathrm{B}$ (Promega), $3 \mathrm{mM} \mathrm{MgCl}_{2}, 0.3 \mathrm{mM}$ each dNTP, 0.4 $\mu \mathrm{M}$ primer L14841, 0.4 $\mu \mathrm{M}$ primer H15149, 1 unit Taq polymerase (Promega) with conditions of preheating at $95^{\circ} \mathrm{C}$ for $5 \mathrm{~min}, 36$ cycles of consecutive incubations at $95^{\circ} \mathrm{C}$ for $30 \mathrm{sec}, 60^{\circ} \mathrm{C}$ for $30 \mathrm{sec}, 72^{\circ} \mathrm{C}$ for $40 \mathrm{sec}$ and $72^{\circ} \mathrm{C}$ for $5 \mathrm{~min}$. A negative control was included for each batch of assay. Amplified DNA products were confirmed with $1.2 \%$ agarose gel and visualized under ultraviolet (UV) light after staining with $2 \mathrm{mg} / \mathrm{mL}$ ethidium bromide. A 100 bp DNA ladder was used as the standard marker for comparison.

The final PCR products of $359 \mathrm{bp}$ were purified using the Exonuclease I/Shrimp Alkaline Phosphatase (GE Healthcare, US) before sequencing by using BigDye Terminator version 3.1 Cycle Sequencing kit (Applied Biosystems, Foster City, CA, USA) and an ABI PRISM 3130 DNA automated sequencer (Applied Biosystems). Sequencing data were analyzed using SeqScape v.2.5 software (Applied Biosystems).

To identify vertebrate host species, the obtained unknown sequences were compared with those already deposited in the GenBank database using BLAST program searches (Basic Local Alignment Search Tool, NCBI. Available online from: http://blast.ncbi.nlm.nih.gov). Sequences of a given pair-wise alignment with the lowest E-value were selected as the most likely species of host.

\section{Results}

Sand fly species diversity and relative abundance A total of 2650 sand flies (1454 females and 1196 males) were collected in this survey from the Louata locality from June to August 2008. Morphological analysis identified 7 Phlebotomus species of three subgenera as follows: P. longicuspis, P. perniciosus, P. langeroni and P. ariasi of the subgenus Larroussius; $P$. sergenti and P. chabaudi of the subgenus Paraphlebotomus; P. papatasi of the subgenus

Table 1 Sand flies collected in the Louata locality: species diversity, relative abundance and infection rates (\%)

\begin{tabular}{lllllll}
\hline Species & Male & Female & Total & $\%$ & Infected sand flies (\%) & Leishmania species \\
\hline P. longicuspis & 764 & 1126 & 1890 & 71.32 & $3(0.26)$ & L. infantum \\
P. sergenti & 281 & 276 & 557 & 21.01 & $4(1.44)$ & L. tropica \\
P. perniciosus & 120 & 15 & 135 & 5.09 & - & - \\
P. papatasi & 18 & 18 & 36 & 1.35 & - & - \\
P. langeroni & 10 & 10 & 20 & 0.75 & - & - \\
P. ariasi & 1 & 4 & 5 & 0.18 & - & - \\
P. chabaudi & 0 & 1 & 1 & 0.03 & - & - \\
S. antennata & 2 & 0 & 2 & 0.07 & - & - \\
S. minuta & 0 & 3 & 3 & 0.11 & - & - \\
S. drefussi & 0 & 1 & 1 & 0.03 & - & - \\
Total & 1196 & 1454 & 2650 & 100 & 7 & - \\
\hline
\end{tabular}


Phlebotomus. Three Sergentomyia species were also identified: Sergentomyia antennata, S. minuta and S. drefussi.

The predominant species in this focus was $P$. longicuspis followed in descending order by $P$. sergenti, $P$. perniciosus, $P$. papatasi, $P$. langeroni, $P$. ariasi, S. antennata, S. minuta, P. chabaudi and S. drefussi (Table 1). In June and August, only 5 Phlebotomus species were collected; whereas, the highest phlebotomine density and diversity was observed in July; all sand fly species were represented, apart from $P$. langeroni (Table 2).

\section{Leishmania infections in sand flies}

Fifty five monospecific pools of female sand flies were screened for Leishmania infection by nested PCR. To identify Leishmania spp., PCR products were directly sequenced using the nested forward primer ITS1F overlapping the 3' end of the SSU rRNA gene and ITS1 with the nested reverse primer ITS2R4 at the 5' end of ITS2. Sequences showed a length range of 390-392 base pairs. Seven pools, 3 P. longicuspis and $4 P$. sergenti were found to be infected by $L$. infantum and $L$. tropica. The seven positive pools were collected from domestic animal shelters and inside houses.

\section{Leishmania DNA sequencing and Neighbor-Joining analysis}

Phylogenetic analysis based on the partial sequence of the ITS-5.8S rDNA using the BLAST program showed that the sequences KJ567478 and KJ567477 were similar to the Iranian L. tropica sequence deposited in GenBank under the accession number JX560482 (99\%), while KJ567476 and KJ567479 were similar to the Moroccan L. tropica sequence deposited in GenBank under the accession number KC145155 (99\% and 97\% respectively).

On the other hand, the sequences KJ567482 and KJ567481 were similar to the Iranian L. infantum sequence deposited in GenBank under the accession number KC477100 (99\% and 96\% respectively), while KJ567480

Table 2 Species and abundance of sand flies collected from June to August in the Louata locality

\begin{tabular}{lllll}
\hline Species & June & July & August & Total \\
\hline P. longicuspis & 444 & 1186 & 260 & 1890 \\
P. sergenti & 174 & 369 & 14 & 557 \\
P. perniciosus & 49 & 68 & 18 & 135 \\
P. papatasi & 10 & 24 & 2 & 36 \\
P. langeroni & 0 & 0 & 20 & 20 \\
P. ariasi & 1 & 4 & 0 & 5 \\
P. chabaudi & 0 & 1 & 0 & 1 \\
S. antennata & 0 & 2 & 0 & 2 \\
S. minuta & 0 & 3 & 0 & 3 \\
Total & 678 & 1658 & 314 & 2650 \\
\hline
\end{tabular}

was similar to the Italian L. infantum sequence deposited in GenBank under the accession number JX945645 (98\%) (Figure 2).

\section{Sand fly blood meal identification}

Ten freshly fed female sand flies (6 P. longicuspis and 4 $P$. sergenti) collected inside houses and in domestic animal shelters were tested by $C y t b$ PCR to determine the blood meal origin. A positive PCR was obtained for all sand flies tested. Negative control yielded no PCR product implying that only host DNA patterns were detected. Direct sequencing of the 10 amplified parts of the Cyt $b$ gene was performed and sequence analysis demonstrated that all sequences were 95\% identical to human sequences.

\section{Discussion}

Phlebotomine sand flies are present in periurban, rural and sylvatic environments and distributed in all countries around the Mediterranean basin. Therefore, human populations and domestic animals living in these areas are potential targets of sand fly-borne diseases, such as leishmaniasis. Knowledge of the distribution of the sand fly population is important in predicting the spatial and temporal variations in the risk of leishmaniasis.

In this work, we report the distribution, Leishmania infection rate and blood feeding preferences of phlebotomine sand flies found in the Louata locality, where sporadic human cases of CL continue to occur every year. Among 23 species described in Morocco, ten sand fly species $(43 \%)$ were identified in this area. July had the highest sand -fly abundance and diversity; $P$. longicuspis was clearly the dominant species, exceeding the cumulative abundance of the other species identified (71.32\%); this species has been incriminated elsewhere in the transmission of L. infantum [28]. The second predominant species identified in this area was P. sergenti $(21.01 \%)$, the confirmed vector of $L$. tropica throughout North Africa, the Middle East and Central Asia [9,29-32].

$P$. sergenti the main vector of $L$. tropica has a wide distribution from sub-Saharan Sahel to the center of Asia through the Middle East and India and is also the most adapted to the Mediterranean climate especially for semi-arid habitats [29].

In Morocco, L. tropica was first isolated from P. sergenti over three decades ago [30] and recently from P. sergenti in support of its vector status, in an emerging CL focus in the Centre of Morocco [3]. In this study, L. tropica DNA was found in four P. sergenti females; the prevalence of infection was almost $1.44 \%$; this rate may be a consequence of a high level of circulation of this Leishmania species in this focus, where cutaneous leishmaniasis is reported to be due to L. tropica [12]. Furthermore, the analysis of the blood meal in the P. sergenti females showed that they all fed on humans. The relative abundance of P. sergenti, 


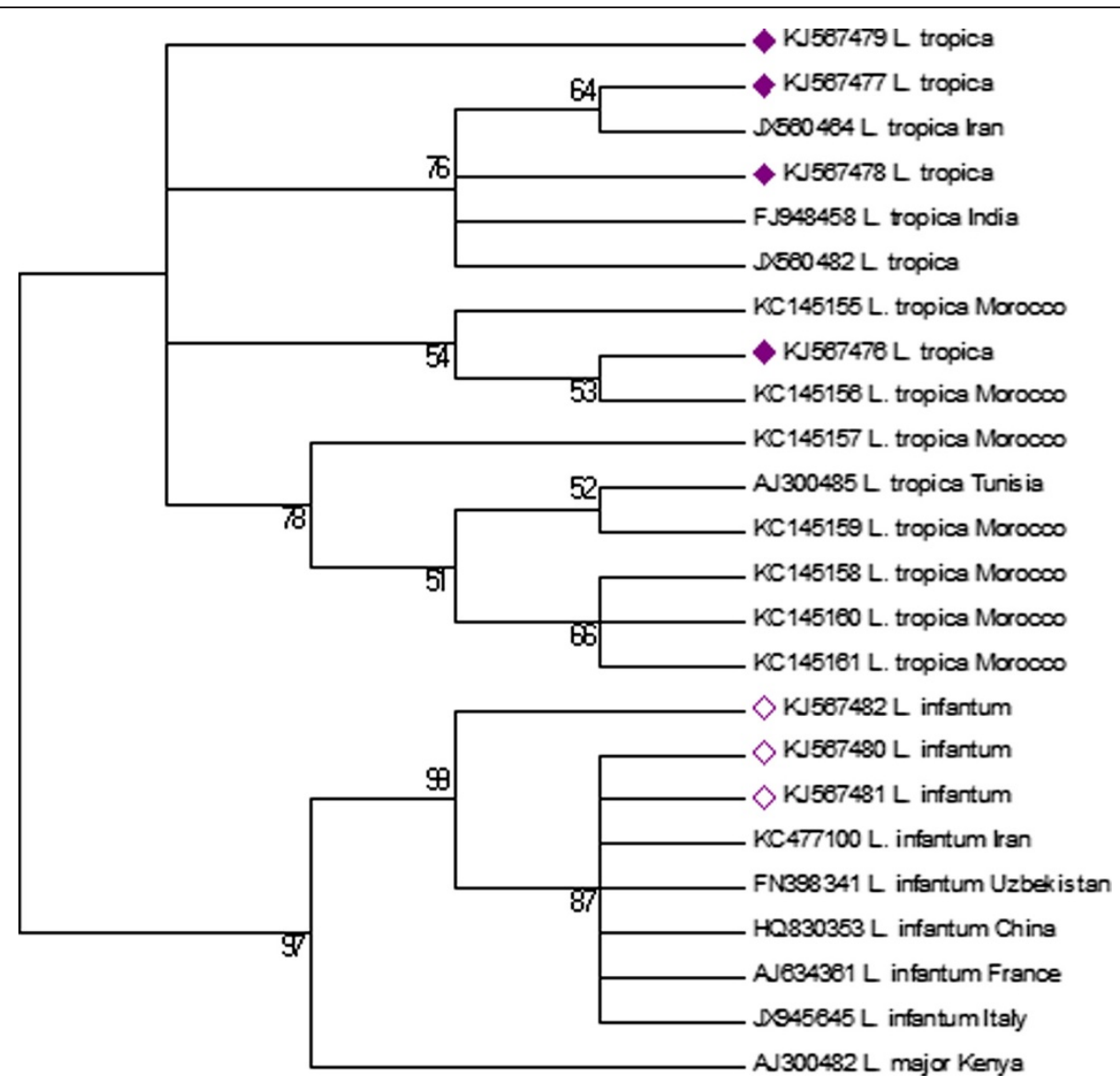

Figure 2 Neighbor-Joining tree showing the relationships of the sequences of L. tropica and L. infantum using MEGA 6 software, related to sequences in GenBank. The evolutionary history was inferred using the Neighbor-Joining method. The optimal tree with the sum of branch length $=0.17897865$ is shown. The percentage of replicate trees in which the associated taxa clustered together in the bootstrap test (1000 replicates) is shown next to the branches. The evolutionary distances were computed using the Maximum Composite Likelihood method and are in the units of the number of base substitutions per site. The analysis involved 24 nucleotide sequences. All positions containing gaps and missing data were eliminated. There were a total of 362 positions in the final dataset. Evolutionary analyses were conducted in MEGA6.

coupled to its anthropophilic character, and its high natural infection rate with L. tropica among unengorged females, constitutes a strong indication that this species plays a major role as the principal, if not the only vector of L. tropica in this area. On the other hand, the screening for L. tropica infection of the other sand fly species separated into monospecific pools showed no positive PCR, suggesting that $P$. sergenti is the only vector of $L$. tropica in this area.

P. longicuspis has been most frequently recorded in the semiarid, arid and periarid Mediterranean bioclimate zones of north-west Africa [2]. In Morocco, Rioux et al. found $P$. longicuspis in all areas, from the sub humid belt to the Sahara, and at varying altitudes [2].

P. longicuspis has been considered an important vector of L. infantum Nicolle in Algeria since the 1940s [33]. In 1990, Rioux and Lanotte proved its role in the transmission of $L$. infantum in a cutaneous leishmaniasis focus [28]. Recently in Algeria, L. infantum DNA was detected in $P$. longicuspis from a visceral leishmaniasis endemic focus [34]. In Morocco, several epidemiological and entomological findings, including the abundance and endophily of this species suggest the capacity of $P$. longicuspis to be a vector; Dereure et al. reported that in the pre-Saharan area, $P$. longicuspis is the only species of the subgenus Larroussius sufficiently abundant to be suspected of transmitting visceral leishmaniasis [35]. In the same way, and based on its abundance, this species is considered to be a suspected vector of $L$. infantum in a focus of leishmaniasis in northern Morocco [11].

In the present work, $P$. longicuspis was the most predominant species (71.32\%), and we report for the first time, both in this focus and in the country, the presence of $L$. infantum DNA within three unengorged females; the results of blood meal analyses by the Cyt $b$ gene indicate that $P$. longicuspis is strongly anthroponotic; since all engorged females contained human blood. So, based on its abundance and anthropophily, in addition to the 
fact that it was the only species found naturally infected with DNA of $L$. infantum, we conclude that $P$. longicuspis can be incriminated as the vector of $L$. infantum in this leishmaniasis focus. This phlebotomine species has never been involved in the transmission of $L$. infantum in Morocco, even if it is a known proven vector elsewhere.

In this work, we applied molecular techniques for the detection of $L$. tropica and $L$. infantum in $P$. sergenti and $P$. longicuspis respectively, as well as in the study of their host preferences. In recent years, molecular techniques have been used increasingly to identify Leishmania infection in phlebotomine sand flies, as a sensitive and effective tool useful in epidemiological studies [16,18,36,37]. The increasing application of molecular techniques in this field has considerably reduced the time needed to obtain results. On the other hand, the use of field collected samples without parasite cultivation is interesting in more ways than one, indeed, previous publications showed evidence that cultivation selects subpopulations of the parasites in the biological samples, including co-existence of nonpathogenic trypanosomatids [38]. In addition, direct analysis of Leishmania DNA in field collected samples produced an unexpected outcome, suggestive of $L$. tropica or L. infantum or a mixture of the two in CL lesions [39]. PCRs based on the amplification of the ITS-5.8S rRNA gene have been used for molecular detection of Leishmania spp. in biological samples and have proved to be highly sensitive [22]. Indeed, this PCR approach has been applied successfully in the detection of Leishmania spp. in entomological surveys $[3,22]$. In addition, we used the $C y t b$ to identify blood meals, which has been widely employed due to its high copy numbers and sufficient genetic variation among vertebrate taxa at the primary sequence level [40-42].

Our results highlight two findings. Firstly, P. longicuspis was more abundant than $P$. sergenti in this cutaneous leishmaniasis focus, where, even if L. tropica was identified in CL [12], it may not be the only species responsible for the disease, as the survey was done only on a small number of skin samples [12]. Secondly, we report for the first time the natural infection of $P$. longicuspis with $L$. infantum in Morocco, which incriminates it as a vector of this parasite. The presence of L. infantum, and its presumed vector in this area, where cutaneous leishmaniasis is already widespread, makes this a site of high risk of visceral leishmaniasis, mostly because of the proximity of a human and canine visceral leishmaniasis focus, less than $20 \mathrm{~km}$ from this investigated area [43]. Furthermore, the absence of VL cases does not mean that this locality is VL free, since most $L$. infantum visceral infections remain asymptomatic. More epidemiological investigations are needed to demonstrate the role of $L$. infantum circulating in this area in the transmission of visceral and/or cutaneous leishmaniasis.

\section{Conclusion}

This focus of leishmaniasis, where $L$. tropica and $L$. infantum co-exist appears peculiar. This result has important implications for drawing attention to the diagnosis and treatment of Leishmania infections. Programs and/or monitoring projects should be developed to detect risk of exposure to mixed infections with Leishmania spp. The follow up of patients with cutaneous leishmaniasis living in this area is necessary in order to reach a better understanding of the interactions between the two parasites.

\section{Competing interests}

Authors declare that they have no competing interests.

\section{Authors' contributions}

NE Carried out the laboratory work of entomological and molecular studies, analyzed data, and prepared the manuscript. MA Contributed to the entomological studies and data analysis. ALI is involved in the study design. $M F$ is involved in the review of the manuscript. ML designed the study, entomological studies, contributed to interpretation, analyzed and finalized the manuscript. All the authors read and approved the final version of the manuscript.

\section{Acknowledgements}

This work was supported by EMRO-COMSTECH grant; RAB \& GH 10-11/09. We would like to thank the team of the Department of Parasitology, Direction d'Epidémiologie et de Lutte Contre les Maladies, Moroccan Ministry of Health. The authors are also very grateful to Doctors and nurses of Sefrou province and the Health representation of the $\mathrm{MOH}$ in Sefrou.

\section{Author details}

'Laboratoire de Parasitologie et Maladies Vectorielles, Institut Pasteur du Maroc, 1 Place Louis Pasteur, 20360 Casablanca, Morocco. ${ }^{2}$ Laboratoire de chimie Bioorganique et analytique, URAC C22, faculté des sciences et techniques, Université Hassan II Mohammedia-Casablanca, Mohammedia, Morocco. ${ }^{3}$ Service de Parasitologie, DELM, Ministère de la Santé, Rabat, Morocco.

Received: 28 March 2014 Accepted: 26 June 2014

Published: 2 July 2014

\section{References}

1. Moroccan Health Ministry: Etat d'avancement des programmes de lutte contre les maladies parasitaires. Direction de l'Épidémiologie et de Lutte Contre les Maladies: Rap ann 2011.

2. Rioux JA, Rispail P, Lanotte G, Lepart J: Phlebotomus bioclimates relations ecology of leishmaniasis epidemiological corollaries: The example of Morocco. Botanical News 1984, 131:549-557.

3. Ajaoud M, Es-sette N, Hamdi S, El-Idrissi AL, Riyad M, Lemrani M: Detection and molecular typing of Leishmania tropica from Phlebotomus sergenti and lesions of cutaneous leishmaniasis in an emerging focus of Morocco. Parasit Vectors 2013, 6:217.

4. Rhajaoui M, Nasereddin A, Fellah H, Azmi K, Amarir F, Al-Jawabreh A, Ereqat S, Planer J, Abdeen Z: New clinico-epidemiologic profile of cutaneous leishmaniasis, Morocco. Emerg Infect Dis 2007, 13:1358-1360.

5. Lemrani M, Nejjar N, Benslimane A: A new focus of cutaneous leishmaniasis due to Leishmania infantum in Northern Morocco. G Ital Med Trop 1999, 4:3-4

6. Rioux JA, Mahjour J, Gallego M, Dereure J, Perieres J, Laamrani A, Riera C, Saddikiand A, Mouki B: Leishmaniose cutanée humaine à Leishmania infantum MON-24 au Maroc. Bull Soc Fr Parasitol 1996, 14:179-183.

7. Nejjar R, Lemrani M, Malki A, Ibrahimy S, Amarouch H, Benslimane A Canine leishmaniasis due to Leishmania infantum MON-1 in northern Morocco. Parasite 1998, 5:325-330.

8. Giorgobiani E, Lawyer PG, Babuadze G, Dolidze N, Jochim RC, Tskhvaradze L, Kikaleishvili K, Kamhawi S: Incrimination of Phlebotomus kandelakii and 
Phlebotomus balcanicus as vectors of Leishmania infantum in Tbilisi, Georgia. PLoS Negl Trop Dis 2012, 6:e1609.

9. Killick-Kendrick R: Phlebotomine vectors of the leishmaniases: a review. Med Vet Entomol 1990, 4:1-24.

10. Ready PD: Leishmaniasis emergence in Europe. Euro Surveill 2010, 15:19505.

11. Guessous-Idrissi N, Hamdani A, Rhalem A, Riyad M, Sahibi H, Dehbi F, Bichichi M, Essari A, Berrag B: Epidemiology of human visceral leishmaniasis in Taounate, a northern province of Morocco. Parasite 1997, 4:181-185.

12. Hmamouch A, Fatima A, Hajiba F, Mbarek K, Khadija B, Mohamed R, Faiza S: Coexistence of Leishmania tropica and Leishmania infantum in Sefrou province, Morocco. Acta Trop 2014, 130C:94-99.

13. Moroccan Health Ministry: Etat d'avancement des programmes de lutte contre les maladies parasitaires. Direction de l'Épidémiologie et de Lutte Contre les Maladies: Rap ann 2008.

14. Amro A, Hamdi S, Lemrani M, Mouna I, Mohammed H, Mostafa S, Rhajaoui M, Hamarsheh O, Schonian G: Moroccan Leishmania infantum: genetic diversity and population structure as revealed by multi-locus microsatellite typing. PLoS One 2013, 8:e77778.

15. Rioux JA, Lanotte G, Petter F, Dereure J, Akalay O, Pratlong F, Velez ID, Fikri NB, Maazoun R, Denial M: Les leishmanioses cutanées du bassin méditerranéen occidental: de l'identification enzymatique à l'analyse éco-épidémiologique. L'exemple de trois "foyers ", tunisien, marocain et français. Leishmania Taxonomie et phylogenèse Applications écoépidémiologiques. (CollintCNRS/INSERM) IMEE, Montpellier 1986, 1986:365-395.

16. Rossi E, Bongiorno G, Ciolli E, Di Muccio T, Scalone A, Gramiccia M, Gradoni L, Maroli M: Seasonal phenology, host-blood feeding preferences and natural Leishmania infection of Phlebotomus perniciosus (Diptera, Psychodidae) in a high-endemic focus of canine leishmaniasis in Rome province, Italy. Acta Trop 2008, 105:158-165.

17. Sharma U, Singh S: Insect vectors of Leishmania: distribution, physiology and their control. J Vector Borne Dis 2008, 45:255-272.

18. Aransay AM, Scoulica E, Tselentis Y: Detection and identification of Leishmania DNA within naturally infected sand flies by seminested PCR on minicircle kinetoplastic DNA. App/ Environ Microbio/ 2000, 66:1933-1938.

19. Perruolo G, Noris Rodriguez N, Feliciangeli MD: Isolation of Leishmania (Viannia) braziliensis from Lutzomyia spinicrassa (species group Verrucarum) Morales Osorno Mesa, Osorno and Hoyos 1969, in the Venezuelan Andean region. Parasite 2006, 13:17-22.

20. Moroccan Health Ministry: Lutte Contre les Leishmanioses. Guide Act 2010.

21. Chomczynski P, Sacchi N: Single-step method of RNA isolation by acid guanidinium thiocyanate-phenol-chloroform extraction. Anal Biochem 1987, 162:156-159.

22. Parvizi P, Mauricio I, Aransay AM, Miles MA, Ready PD: First detection of Leishmania major in peridomestic Phlebotomus papatasi from Isfahan province, Iran: comparison of nested PCR of nuclear ITS ribosomal DNA and semi-nested PCR of minicircle kinetoplast DNA. Acta Trop 2005, 93:75-83.

23. Parvizi P, Ready PD: Nested PCRs and sequencing of nuclear ITS-rDNA fragments detect three Leishmania species of gerbils in sandflies from Iranian foci of zoonotic cutaneous leishmaniasis. Trop Med Int Health 2008, 13:1159-1171.

24. Tamura K, Stecher G, Peterson D, Filipski A, Kumar S: MEGA6: Molecular Evolutionary Genetics Analysis version 6.0. Mol Biol Evol 2013, 30:2725-2729.

25. Saitou NaN M: The neighbor-joining method: A new method for reconstructing phylogenetic trees. Mol Biol Evol 1987, 4:406-425.

26. Felsenstein J: Confidence limits on phylogenies: An approach using the bootstrap. Evolution 1985, 39:783-791.

27. Boakye DA, Tang J, Truc P, Merriweather A, Unnasch TR: Identification of bloodmeals in haematophagous Diptera by cytochrome B heteroduplex analysis. Med Vet Entomol 1999, 13:282-287.

28. Rioux JA, Lanotte G: Leishmania infantum as a cause of cutaneous leishmaniasis. Trans R Soc Trop Med Hyg 1990, 84:898.

29. Al-Zahrani MA, Peters W, Evans DA, Chin C, Smith V, Lane RP: Phlebotomus sergenti, a vector of Leishmania tropica in Saudi Arabia. Trans $R$ Soc Trop Med Hyg 1988, 82:416.

30. Guilvard E, Rioux JA, Gallego M, Pratlong F, Mahjour J, Martinez-Ortega E, Dereure J, Saddiki A, Martini A: Leishmania tropica au Maroc. III. Rôle de Phlebotomus sergenti. A propros de 89 isolats. Ann Parasitol Hum Comp 1991, 66:96-99.
31. Boubidi SC1, Benallal K, Boudrissa A, Bouiba L, Bouchareb B, Garni R, Bouratbine A, Ravel C, Dvorak V, Votypka J, Volf P, Harrat Z: Phlebotomus sergenti (Parrot, 1917) identified as Leishmania killicki host in Ghardaia, south Algeria. Microbes Infect 2011, 13:691-696.

32. Tabbabi A, Bousslimi N, Rhim A, Aoun K, Bouratbine A: First report on natural infection of Phlebotomus sergenti with Leishmania promastigotes in the cutaneous leishmaniasis focus in southeastern Tunisia. Am J Trop Med Hyg 2011, 85:646-647.

33. Parrot $L$, Donatien $A$ : [New observations on the natural infection of Phlebotomus by general leishmaniasis of man and dog in Algeria]. Arch Inst Pasteur Alger 1952, 30:146-152.

34. Harrat Z, Belkaid M: Leishmaniasis in Algiers: epidemiologic data. Bull SoC Pathol Exot 2003, 96:212-214

35. Dereure J, Velez ID, Pratlong F, Denial M, Lardi M, Moreno G, Serres E, Lanotte G, Rioux JP: La leishmaniose viscerale autochtone au Maroc méeridional. Présence de Leishmania infantum MON-1 chez le Chien en zone presaharienne. Leishmania Taxonomie et Phylogenese Applications éco- épidémiologiques. (Coll Int CNRS/INSERM) 1984, 1986:421-425.

36. Kato $H$, Uezato $H$, Katakura K, Calvopiña M, Marco JD, Barroso PA, Gomez EA, Mimori T, Korenaga M, Iwata H, Nonaka S, Hashiguchi Y: Detection and identification of Leishmania species within naturally infected sand flies in the andean areas of ecuador by a polymerase chain reaction. Am J Trop Med Hyg 2005, 72:87-93.

37. Scarpassa VM, Alencar RB: Molecular taxonomy of the two Leishmania vectors Lutzomyia umbratilis and Lutzomyia anduzei (Diptera: Psychodidae) from the Brazilian Amazon. Parasit Vectors 2013, 6:258

38. Singh N, Chikara S, Sundar S: SOLiD ${ }^{\text {TM }}$ sequencing of genomes of clinical isolates of Leishmania donovani from India confirm leptomonas co-infection and raise some key questions. PLoS One 2013, 8:e55738.

39. Toz SO, Culha G, Zeyrek FY, Ertabaklar H, Alkan MZ, Vardarli AT, Gunduz C, Ozbel Y: A real-time ITS1-PCR based method in the diagnosis and species identification of Leishmania parasite from human and dog clinical samples in Turkey. PLoS Negl Trop Dis 2013, 7:e2205.

40. Castresana J: Cytochrome b phylogeny and the taxonomy of great apes and mammals. Mol Biol Evol 2001, 18:465-471.

41. Ngo KA, Kramer LD: Identification of mosquito bloodmeals using polymerase chain reaction (PCR) with order-specific primers. J Med Entomol 2003, 40:215-222.

42. Yang BB, Chen DL, Chen JP, Liao L, Hu XS, Xu JN: Analysis of kinetoplast cytochrome $b$ gene of 16 Leishmania isolates from different foci of China: different species of Leishmania in China and their phylogenetic inference. Parasit Vectors 2013, 6:32

43. Nejjar R, Lemrani M, Amarouche A, Benslimane A: Variation in antibody titres against Leishmania infantum in naturally infected dogs in northern Morocco. Rev Med Vet 2000, 151:841-846.

\section{doi:10.1186/1756-3305-7-305}

Cite this article as: Es-Sette et al:: Molecular detection and identification of Leishmania infection in naturally infected sand flies in a focus of cutaneous leishmaniasis in northern Morocco. Parasites \& Vectors 2014 7:305.

\section{Submit your next manuscript to BioMed Central and take full advantage of:}

- Convenient online submission

- Thorough peer review

- No space constraints or color figure charges

- Immediate publication on acceptance

- Inclusion in PubMed, CAS, Scopus and Google Scholar

- Research which is freely available for redistribution 